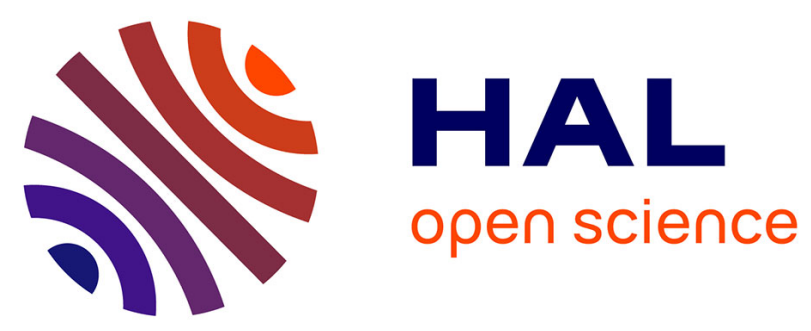

\title{
What can we learn from the fission time of the super-heavy elements?
}

D. Boilley, A. Marchix, D. Wilgenbus, Y. Lallouet, F. Gimbert, Y. Abe

\section{To cite this version:}

D. Boilley, A. Marchix, D. Wilgenbus, Y. Lallouet, F. Gimbert, et al.. What can we learn from the fission time of the super-heavy elements?. International Workshop on Nuclear dynamics in Heavy-Ion Reactions and Neutron Stars, Jul 2007, Beijing, China. pp.1681-1693, 10.1142/S0218301308010696 . in2p3-00311013

\section{HAL Id: in2p3-00311013 https://hal.in2p3.fr/in2p3-00311013}

Submitted on 12 Aug 2008

HAL is a multi-disciplinary open access archive for the deposit and dissemination of scientific research documents, whether they are published or not. The documents may come from teaching and research institutions in France or abroad, or from public or private research centers.
L'archive ouverte pluridisciplinaire HAL, est destinée au dépôt et à la diffusion de documents scientifiques de niveau recherche, publiés ou non, émanant des établissements d'enseignement et de recherche français ou étrangers, des laboratoires publics ou privés. 
What can we learn from the fission time of the super-heavy elements?

\author{
David BOILLEY; Anthony MARCHIX, David WILGENBUS, Yoann LALLOUET, Florian \\ GIMBERT \\ GANIL, CEA/DSM-CNRS/IN2P3, BP 55027, F-14076 Caen cedex 5, France \\ boilley@ganil.fr \\ Yasuhisa ABE
}

RCNP, Osaka University, 10-1 Mihogaoka, Ibaraki, Osaka, 567-0047, Japan

Received (received date)

Revised (revised date)

\begin{abstract}
Recent experiments performed at GANIL with a crystal blocking technique have shown direct evidences of long fission times in the Super-Heavy Elements (SHE) region. Aimed to localize the SHE island of stability, can these experiments give access to the fission barrier and then to the shell-correction energy? In this paper, we calculate the fission time of heavy elements by using a new code, KEWPIE2, devoted to the study of the SHE. We also investigate the effect of potential structure beyond the saddle on the fission time.
\end{abstract}

\title{
1. Introduction
}

Considering the bulk properties of the nuclei based on the liquid drop model, the Coulomb part which is proportional to the volume rapidly dominates when the size of the nucleus grows. This leads to a limitation of the size of the nuclei that become unstable when they are deformed, leading to the fission. But, the extrapolation of the shell-model based on stable nuclei leads to the prediction of the existence of Super-Heavy Elements (SHE) that should have gained an extra-stability by the shell closure. They have never been observed in nature. Several heavy-ion facilities in the world have attempted to synthetize them by fusion reaction but are facing the problem of very low probabilities. The island of stability has not been reached so far.

The prediction of the location of the island of stability depends on the theoretical model used. There have been tentatives at GANIL to locate the SHE island by measuring the fission time. ${ }^{1,2,3}$ Since no evaporation residue is expected, the bombarding energy could be higher and the statistic is no longer a problem. Using crystal blocking techniques, direct evidences for very long fission times of $Z=120$ 
and 124 have been shown. But no hint for long lifetimes for the $\mathrm{Z}=114$ case. By long fission times, the authors mean times longer than $10^{-18} \mathrm{~s}$. Do these results mean that $\mathrm{Z}=120$ and 124 elements have a very high fission barrier, and then might be candidates for being metastable SHE?

In this paper, we will present some theoretical calculations of the fission time by a new code, KEWPIE2, which is a statistical and dynamical code devoted to the SHE. ${ }^{4,5}$ There are still many ambiguities in the reaction mechanism that leads to the observed SHE. The fission time measurements give new constraints to the models that are very useful. We will present also some preliminary results on the effect of a second potential pocket on the fission time.

\section{Fission time of a cascade chain}

The fission of hot nuclei is generally seen as a slow collective motion passing over a potential barrier with the help of thermal fluctuations. A Langevin equation ${ }^{6}$, or its Klein-Kramers ${ }^{7,8}$ equivalent, is then used for the formalism. See Refs. ${ }^{9,10,11}$ for reviews. But the very long fission times observed in the crystal blocking experiments cannot be calculated by this formalism that is too much computer-time consuming.

Another approach based on Bateman ${ }^{12}$ equations which are master equations, allows to evaluate long fission times. For the sake of simplicity, we will write the equations with only the evaporation of neutrons. The population of each isotope, $P_{i}(t)$ is governed by

$$
\begin{aligned}
\frac{d P_{0}}{d t} & =-\left(\Gamma_{n, 0}+\Gamma_{f, 0}\right) P_{0}(t) \\
\vdots & =\vdots \\
\frac{d P_{i}}{d t} & =-\left(\Gamma_{n, i}+\Gamma_{f, i}\right) P_{i}(t)+\Gamma_{n, i-1} P_{i-1}(t),
\end{aligned}
$$

where the $\Gamma_{n, i}$ 's and $\Gamma_{f, i}$ 's are respectively the neutron-evaporation widths and fission widths of $i$ th isotope. For the latter, we adopt the Bohr and Wheeler formula ${ }^{13}$ including Strutinsky ${ }^{14}$ and Kramers ${ }^{8}$ corrections, and for the former we use the formalism of Weisskopf. ${ }^{15}$ Charged particle evaporation can easily be included in a similar scheme. ${ }^{4}$

The average fission time is defined as,

$$
\begin{aligned}
\tau_{\text {fiss }} & =-\frac{1}{P_{\text {sum }}(0)-P_{\text {sum }}(\infty)} \int_{0}^{\infty} t \frac{d P_{\text {sum }}}{d t} d t \\
& =\frac{1}{P_{\text {sum }}(0)-P_{\text {sum }}(\infty)} \int_{0}^{\infty} t \sum_{i} \Gamma_{f, i} P_{i}(t) d t,
\end{aligned}
$$

where $P_{\text {sum }}(t)=\sum_{i} P_{i}(t)$. The fission time distribution is then given by $-\frac{d P_{\text {sum }}}{d t}$.

With some simplifications, Bateman equations can easily be solved analytically. 


\subsection{Fission time with a toy model}

Restricting the evaporation mechanism to neutrons and assuming that each neutron takes out its binding energy $B_{n}$ and the kinetic energy equal to its average value $2 T$, Bateman equations can be solved analytically by using the formalism of the Laplace transform. From the expressions of the populations $P_{i}(t)$,

$$
\begin{aligned}
P_{0}(t)= & N_{0} e^{-\left(\Gamma_{n, 0}+\Gamma_{f, 0}\right) t} \\
P_{i}(t)= & N_{i} e^{-\left(\Gamma_{n, i}+\Gamma_{f, i}\right) t} \\
& +\sum_{k=0}^{i-1}\left[\prod_{l=k}^{i-1} \frac{\Gamma_{n, l}}{\Gamma_{n, l}+\Gamma_{f, l}-\left(\Gamma_{n, i}+\Gamma_{f, i}\right)}\left(N_{k} e^{-\left(\Gamma_{n, i}+\Gamma_{f, i}\right) t}-P_{k}(t)\right)\right]
\end{aligned}
$$

it is very easy to evaluate any observable such as the average fission time or the pre-scission neutron multiplicities. ${ }^{16,4}$ The previous eqs. are written in a recursive form, because it is easier to compute. $N_{i}$ is the initial number of each isotope. Here, we took $N_{0}=1$ and 0 for the others.

As a toy model, we will consider that the fission barrier, $B_{f}$ and the neutron binding energy $B_{n}$ are the same for all the isotopes of the evaporation chain. The average fission time is plotted in Fig. 1 as a function of $B_{f} / B_{n}$ for an excitation energy $E^{*}=70 \mathrm{MeV}$ which corresponds to the experimental value. It can be seen that the fission time can become very large, far larger than the inverse of the fission width when $B_{f} \simeq B_{n}$. When $B_{f} \ll B_{n}$, the fission channel dominates, few neutrons are evaporated and the fission time is short. On the contrary, when $B_{f} \gg B_{n}$, the fission events are very rare and only first chance fission contributes to the average. After having evaporated few neutrons, the nuclei are too cold to undergo fission. The long time fission observed experimentally are then due to fission events at the end of a long evaporation chain.

The observation of long fission-times would then mean that the fission barriers should be close to the neutron binding energy all along the evaporation chain. The average fission time does not depend only on the fission barrier of the compound nucleus formed by the fusion reaction, but also on the fission barrier of all the isotopes obtained by the evaporation of neutrons. This will make the analysis of the experimental data more complicated.

Actually, in Refs. ${ }^{1,2,3}$, the authors do not measure the average fission time. They just state that about $10 \%$ of the capture events of the SHE should have a fission-time longer than $10^{-18} \mathrm{~s}$ in order to explain the experimental observations. Here, we will only consider the fission events. In Fig. 2 we plot the fission-time distribution for three typical fission barriers. It appears again that for a low fission barrier $\left(B_{f}=0.2 B_{n}=1.2 \mathrm{MeV}\right)$, there's almost no event with long fission time. With $B_{f}=B_{N} / 2=3 \mathrm{MeV}$, about $0.3 \%$ of the events have a fission time longer than $10^{-18} \mathrm{~s}$, even if the average fission time is $1.4 \times 10^{-19} \mathrm{~s}$ for this simple model. When $B_{f}=B_{n}=6 \mathrm{MeV}$, one finds a long tail for the fission time distribution, as expected. The average fission time is very large, $2 \times 10^{-15} \mathrm{~s}$. It also appears from this simple model that at least 3-4 neutrons should be evaporated before having a 

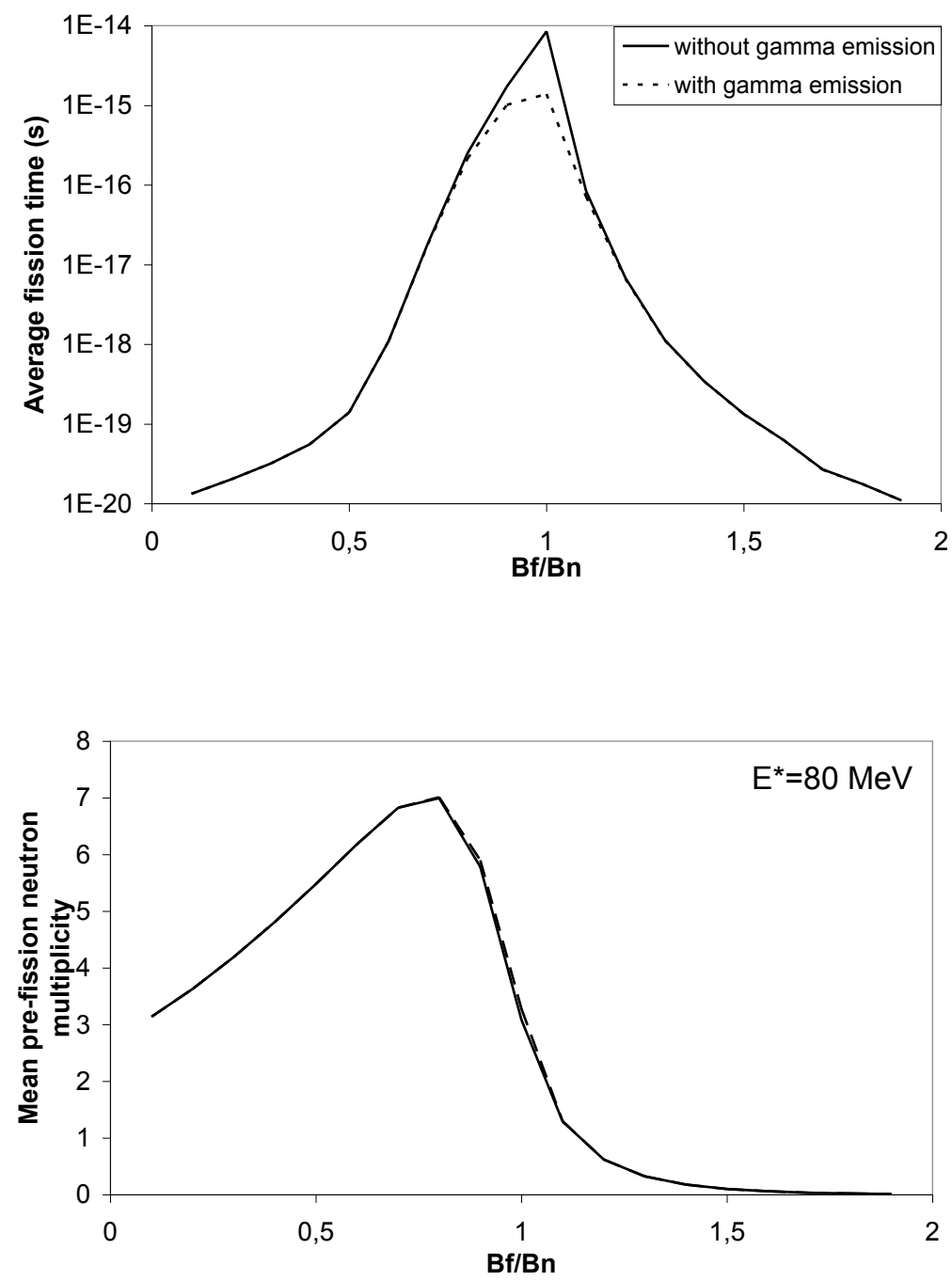

Fig. 1. Top: Average fission time of a $\mathrm{Z}=124$ like nucleus at $E^{*}=70 \mathrm{MeV}$ with the fission barrier, $B_{f}$ and the binding energy of the neutrons $B_{n}$ the same all along the isotope chain. Bottom: pre-scission neutron multiplicities for the same system.

fission time longer than $10^{-18} \mathrm{~s}$. 


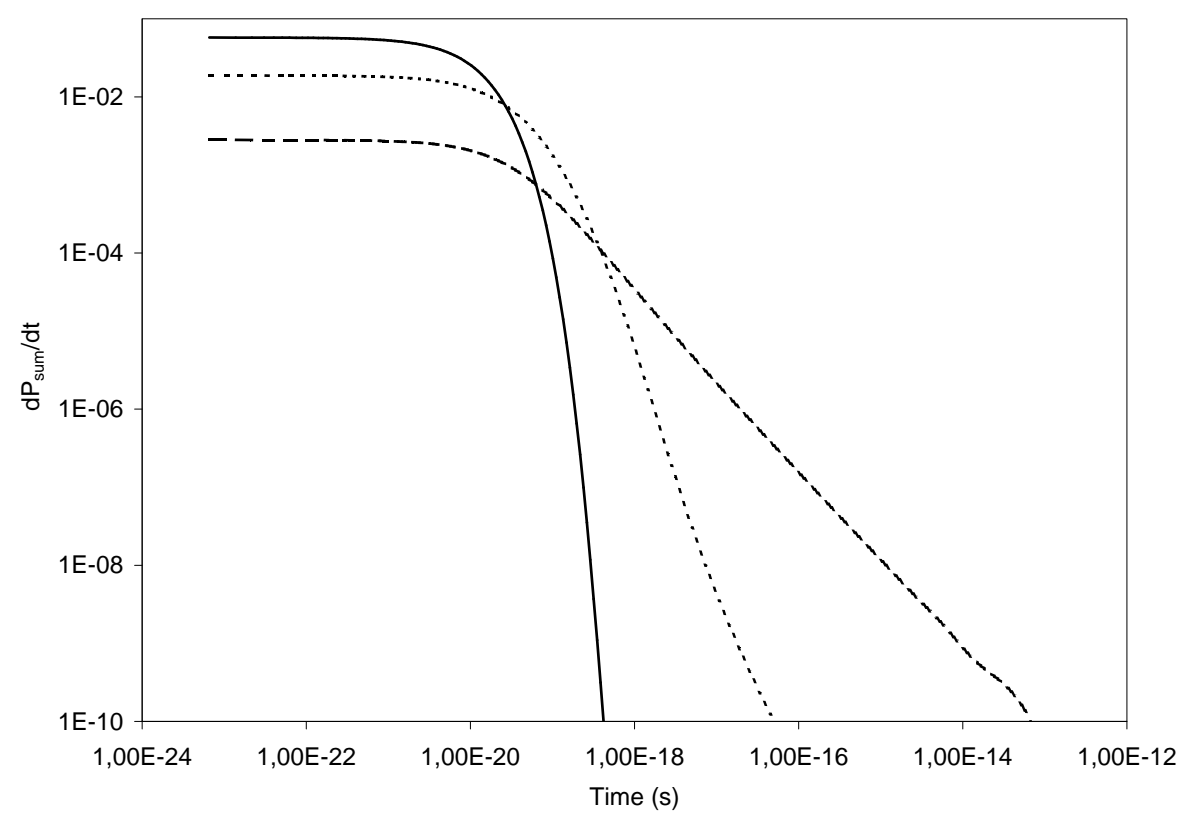

Fig. 2. Time distribution of the fission events as a function of time for the same model as in fig. 1 for various values of the fission-barrier $B_{f}$. The solid line corresponds to $B_{f}=0.2 B_{n}=1.2 \mathrm{MeV}$, the dotted one to $B_{f}=B_{N} / 2=3 \mathrm{MeV}$ and the dashed one to $B_{f}=B_{n}=6 \mathrm{MeV}$.

\subsection{The KEWPIE2 code}

In KEWPIE2 $2^{4,5}$ the Bateman equations are discretized and solved numerically. As the code is mainly devoted to the SHE, Monte Carlo methods are avoided. The energy distribution of each nucleus is divided into bins and evolves in time accordingly to the evaporation process and the energy spectra of the evaporated particles.

As the statistical part is generally considered as the better known part of the reaction mechanism that leads to the SHE, there is no free parameter. Nevertheless, some parameters that are not accurately known can change drasticaly the final results. The shell correction energy is one of these key parameters. The code has been compared to the experimental residue cross sections in the SHE region. ${ }^{19,20}$ When it is applied to the synthesis of heavy elements, for reactions without fusion hindrance, the cross sections are well reproduced. But, in the super-heavy region, if one fits the fusion model on the experimental data, the calculated cross sections generally over-estimate the experimental results. Considering that the experimental fusion probabilities are correct, the theoretical shell correction energy ${ }^{17}$ has to be reduced to fit the residue cross sections. ${ }^{20}$

In this paper, the mass table of $\mathrm{M} \varnothing l$ ler and $\mathrm{Nix}^{17}$ is adopted without any correction. The shell correction energy $\Delta E_{\text {shell }}$ is supposed to be damped when the 


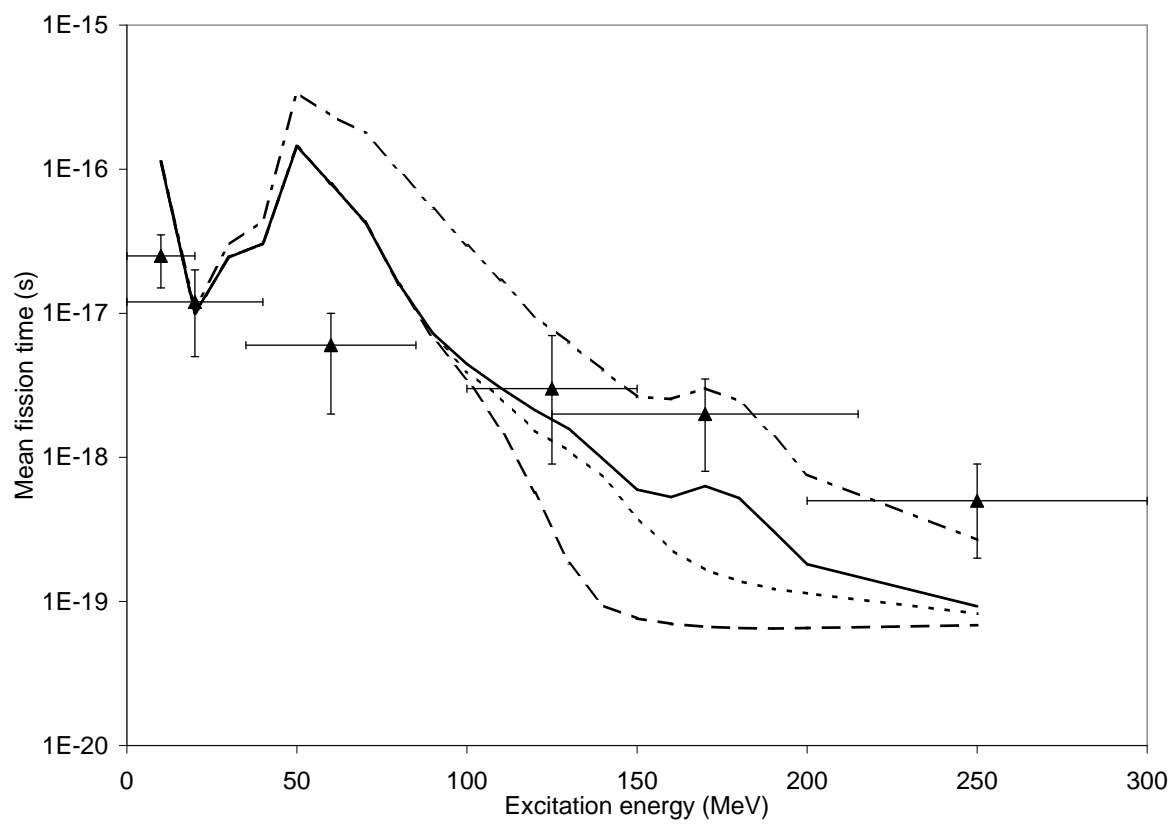

Fig. 3. Average fission time of Uranium. The experimental data were obtained by crystal blocking technique. ${ }^{21}$ The average fission time was calculated with neutron evaporation only (dashed line), with neutron and proton evaporation (dotted line) and neutron, proton and alpha evaporation (solid line). $\gamma$-ray emission is also always included. The reduced friction coefficient is fixed to $\beta=2 \times 10^{21} \mathrm{~s}^{-1}$ for these three curves. The dashed-dotted line corresponds to the average fission time with neutron, proton and alpha evaporation and $\beta=5 \times 10^{21} \mathrm{~s}^{-1}$.

temperature increases according to Ignatyuk ${ }^{18}$ formula for the level density parameter at the ground state,

$$
a_{\text {ground }}=a\left(1+\frac{\left(1-e^{-E^{*} / E_{d}}\right) \Delta E_{\text {shell }}}{E^{*}}\right),
$$

where $E^{*}$ is the excitation energy and $E_{d}$ is the damping energy, a parameter originally set to $E_{d}=18.5 \mathrm{MeV}$.

Finally, the reduced friction coefficient of Kramers is set to $\beta=2 \times 10^{21} \mathrm{~s}^{-1}$, as explained in the next subsection. All the ingredients are naturally the same for the statistical and dynamical parts of the code.

\subsection{Average fission time of Uranium}

The results of the fission of Uranium ${ }^{21}$ also obtained by crystal blocking techniques are used to test the validity of the dynamical part of the KEWPIE2 code, see Fig. 3.

It appears that the charged particles should not be neglected for an excitation energy larger than $100 \mathrm{MeV}$. In this study, we will neglect them since the excitation 


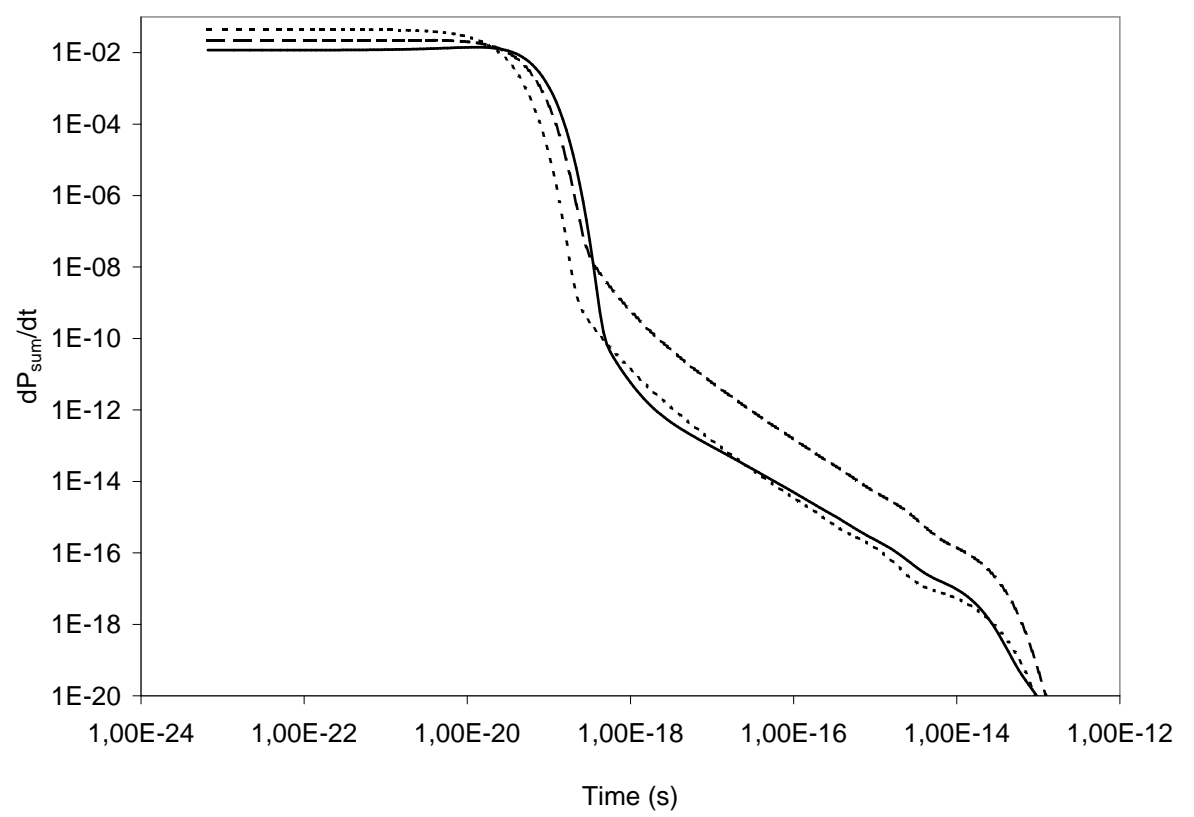

Fig. 4. Fission-time distribution of the $\mathrm{Z}=114$ (dotted line), 120 (dashed line) and 124 (solid line) nuclei with fission barrier $B_{f}$ taken from the table of Møller and Nix. ${ }^{17}$ The damping energy is fixed at $E_{d}=18.5 \mathrm{MeV}$ and $\beta=2 \times 10^{21} \mathrm{~s}^{-1}$.

energy is smaller. For an excitation energy lower than $50 \mathrm{MeV}$, there is a large disagreement between theory and experiment. This is probably due to the fact that quantum effect that are not included in this code should be taken into account at low excitation energy. At about $70 \mathrm{MeV}$ of excitation energy, there is no problem. The average fission time is also very sensitive to the reduced friction parameter which is not well known. ${ }^{9}$ At high excitation energy, $\beta=5 \times 10^{21} \mathrm{~s}^{-1}$ seems to fit better the data. But at around $E^{*}=70 \mathrm{MeV}, \beta=2 \times 10^{21} \mathrm{~s}^{-1}$ appears to be better.

\section{Fission time of the SHE}

The KEWPIE2 code is now directly applied to the fission time of the $Z=114,120$ and 124 SHE, see Fig. 4. In this part, we only take into account the evaporation of neutrons and $\gamma$-ray emission because charged particles do not change the results. The shape of the fission time distributions is not the same as the one obtained with the toy model because of the damping of the shell correction energy that reduces significatively the fission barriers at the beginning of the chain and then enhance the fission channel. At $70 \mathrm{MeV}$ of excitation energy, the fission barriers of the head of the evaporation chain have almost vanished, and all the curves have the same peak. Only the long-time tail differs. 


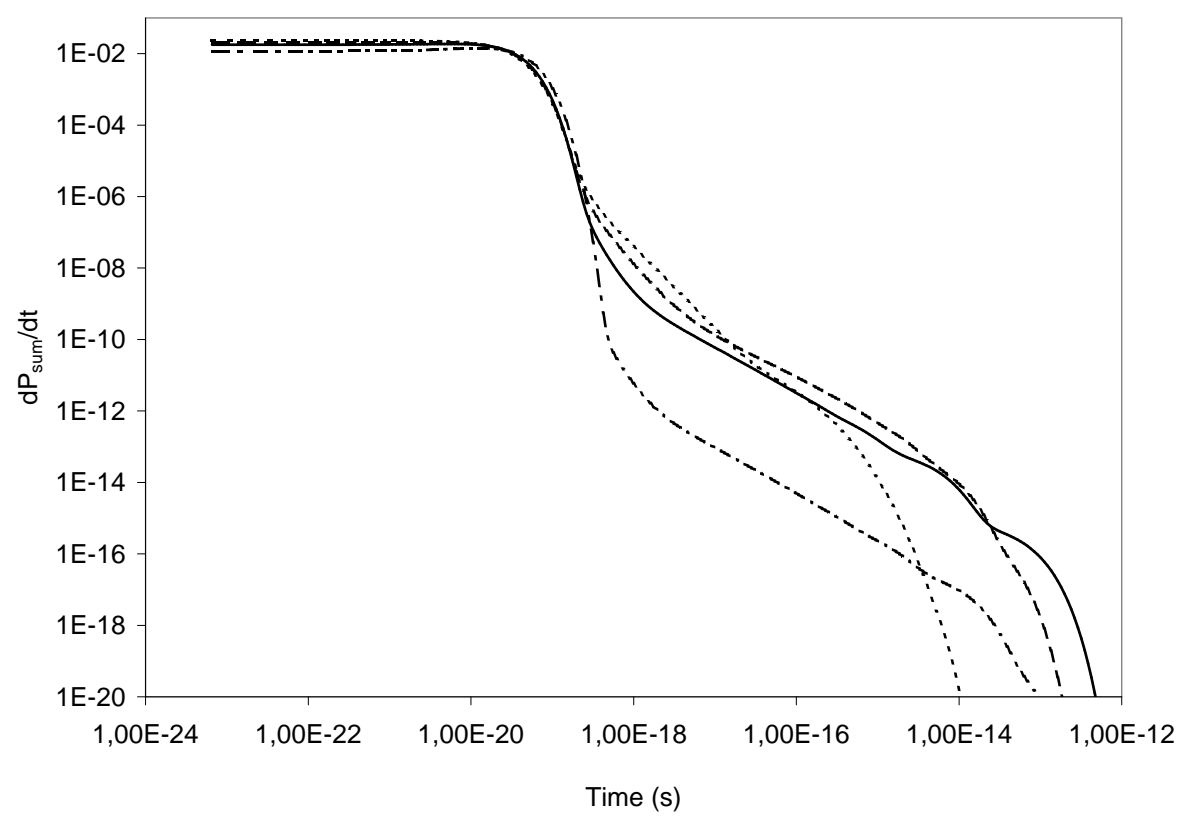

Fig. 5. Fission time distribution of a $\mathrm{Z}=124$ like nucleus with various abitrary and realistic values (dashed-dotted line) of the fission barrier $B_{f}$. Solid line: $B_{f}=6 \mathrm{MeV}$, dashed line: $B_{f}=8 \mathrm{MeV}$ and dotted line: $B_{f}=10 \mathrm{MeV}$.

The $Z=120$ element has the largest long-time fission tail but only $7 \times 10^{-5} \%$ of the events have a fission time longer than $10^{-18} \mathrm{~s}$, which far lower than the $10 \%$ observed experimentally. The situation is worse for the $Z=124$ element for which $9 \times 10^{-7 \%}$ of the events have a long fission time. Finally, the $Z=114$ element has the smallest long-time fission tail which is not contradictory with the experimental results.

For the $Z=120$ element, the long time limit should be reduced by a factor 14 down to $7 \times 10^{-20} \mathrm{~s}$ to have $10 \%$ of the events with a long fission time. This factor is 20 for the $Z=124$ element. This is far lower than the experimental uncertainty on the long time limit.

To reach the experimental statistics, the fission barrier at the beginning of the chain should be far larger or should not be damped. In Fig. 5, we plot the fission time distribution for a $Z=124$ like element with a fission barrier arbitrarily fixed to a constant value all along the chain. With $B_{f}=6 \mathrm{MeV}$ the average fission time is $\tau=3.3 \times 10^{-20} \mathrm{~s}$ and there are only $5 \times 10^{-4 \%}$ of the events with a fission time longer than $10^{-18} \mathrm{~s}$. When it is fixed to $B_{f}=8 \mathrm{MeV}$, the average fission time is almost unchanged, but there $1.8 \times 10^{-3} \%$ events with a long fission time. The tail is shorter but there are more events near $10^{-18} \mathrm{~s}$. This tendency is confirmed when the fission barrier is fixed to $B_{f}=10 \mathrm{MeV}$ all along the chain. The average fission 
time is then $\tau=2.6 \times 10^{-20} \mathrm{~s}$ and $4 \times 10^{-3} \%$ of the events correspond to long fission times. To enlarge the statistics of long fission events, one should have a very large fission barrier at the beginning of the chain to compensate the damping, and have a fission barrier closer to the binding energy of the neutron at the end of the chain. With $B_{f}=12 \mathrm{MeV}$ for the five first elements of the chain, $B_{f}=10 \mathrm{MeV}$ for the next two, and $B_{f}=7 \mathrm{MeV}$ for the last ones, there are $1.6 \%$ of the events with a fission time longer than $10^{-18} \mathrm{~s}$, and the average fission time is $\tau=10^{-17}$ s. This gives an idea of what is necessary to get the experimental statistics.

It appears clearly that the shell correction energy of the table of Møller and $\mathrm{Nix}^{17}$ is not compatible with the data obtained for the $Z=120$ and 124 elements. Of course, one could refine the model. We could consider another damping function, assuming that for a closed shell nuclei, the shell correction energy can better resist to the temperature. Or consider that the friction coefficient depends on the excitation energy. But these refinements might not be able to increase the number of longtime events by orders of magnitude. In the following, we study the influence of the saddle-to-scission time, especially in the presence of isomeric potential pockets.

\section{Influence of the isomeric potential pocket on the fission time}

\subsection{Saddle-to-scission time}

In KEWPIE2, the fission time is calculated at the saddle. But the saddle point of the SHE is very close to the spherical shape of the excited ground state. This means that the scission point is very far and we might not be able to neglect the descend time from the saddle to the scission point. To evaluate the average saddle-to-scission time we use a new formula ${ }^{22}$ that can be applied to any kind of potential.

In Fig. 6 we compare the saddle-to-scission time to the inverse of the fission width at an excitation energy of $70 \mathrm{MeV}$. For the potential between these two points, we are using an analytical formula fitted on LDM potential map in the SHE region of ref. ${ }^{23}$ The saddle-to-scission time is less than an order of magnitude smaller that the usual fission time for large fissibility and cannot be neglected. But it cannot be the clue to explain the large fission time observed.

But if there are some potential pockets beyond the saddle, this would enlarge far more the average fission time. We will investigate this aspect in the next section.

\subsection{Pure fission time with potential having barrier structures}

If the potential has another pocket beyond the saddle, the deformed nucleus could have a life time as long as the nucleus in its fundamental state. To investigate the influence of such a structure on the fission time, we studied various kinds of arbitrary potential. A typical example is shown in Fig. 7.

The fission time is here defined by the average passage-time over the last barrier 
10 D. Boilley, A. Marchix, D. Wilgenbus, Y. Lallouet, F. Gimbert, Y. Abe

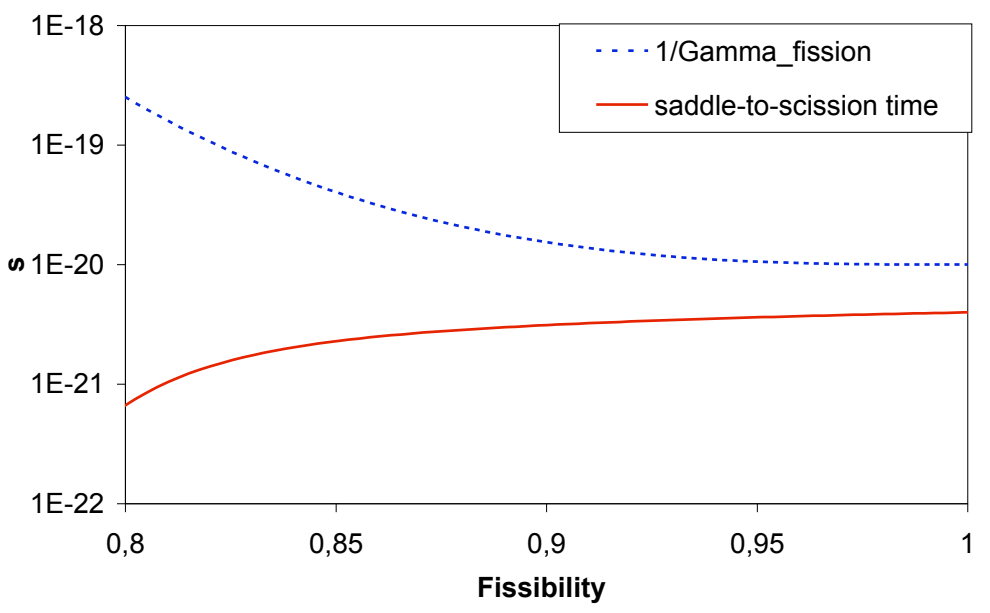

Fig. 6. Inverse of the fission width and saddle-to-scission time as a function of the fissibility for large nuclei. The calculation is done for $E^{*}=70 \mathrm{MeV}$.

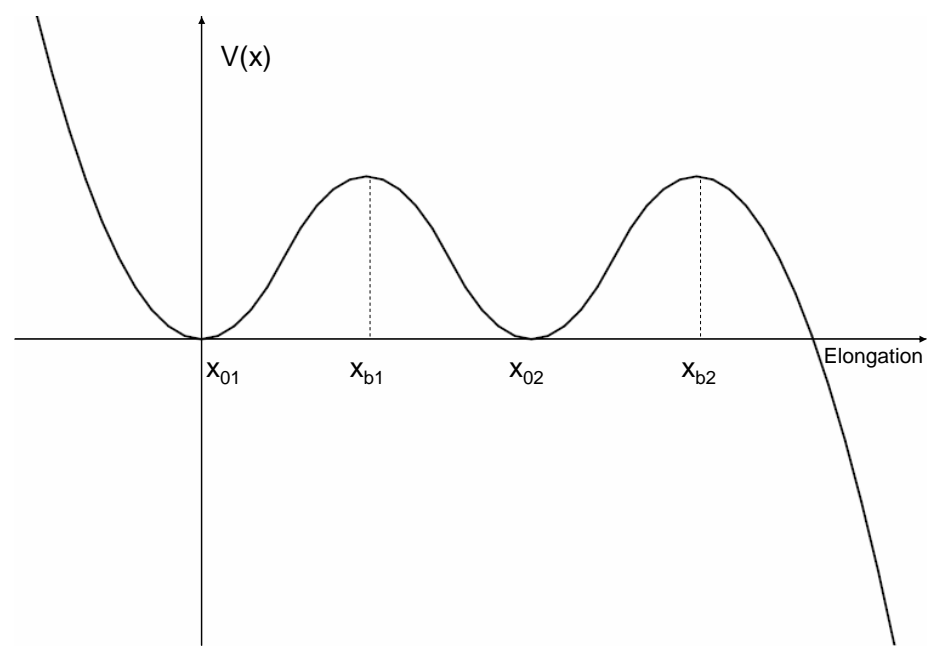

Fig. 7. A typical example of a double-humped potential. 
and can be studied by using the so-called Non Linear Relaxation Time ${ }^{24}$

$$
N L R T=\frac{\int_{0}^{+\infty} P(t) d t}{P(0)-P(\infty)},
$$

for which an analytical formula exists. ${ }^{25}$ In this equation, $P(t)$ is the number of compound nuclei at time $t$ including both fondamental and isomeric states. With such a formalism, we can show that for the potential of Fig. 7, the fission time is about 3 times larger than with a single barrier. For other kinds of double-humped potentials, this factor is lower than 3 .

Since the analytical formula for the NLRT is only valid for very large friction, but any value of the temperature, we checked these results by other means. Considering the double-well problem and calling $\Gamma_{1 \rightarrow 2}, \Gamma_{2 \rightarrow 1}$ and $\Gamma_{2 \rightarrow e}$, the Kramers escape rates over the barriers, we can easily obtain that the average fission time reads

$$
\tau_{f}=\tau_{r}+\frac{1}{\Gamma_{2 \rightarrow e}}\left(1+\frac{\Gamma_{2 \rightarrow 1}+\Gamma_{2 \rightarrow e}}{\Gamma_{1 \rightarrow 2}}\right),
$$

where $\tau_{r}$ is the transient time necessary to reach Kramers stationary limit. Here, we have asumed that the transient function is well approximated by a step function, $\Gamma_{1 \rightarrow 2}(t)=\theta\left(t-\tau_{r}\right) \Gamma_{1 \rightarrow 2}$. The other two widths $\Gamma_{2 \rightarrow 1}$ and $\Gamma_{2 \rightarrow e}$ are supposed to be constant. It is then obvious than we get results similar to the ones obtained with the NLRT. In this approach, the validity of the results of eq. (9) is governed by Kramers approximation: low temperature, but medium to large friction.

These results were also confirmed by numerical simulations with a Langevin equation.

Isomeric potential pockets can then enlarge the fission time quite significatively and can have a significative effect on the statistics of long fission-time events. These results are only valid for pure fission. What is the effect of the evaporation of neutrons on these conclusions?

\subsection{Back to the toy model with a double-humped potential}

Considering the case of a potential having the shape of Fig. 7, we will study the influence of the evaporation of neutrons on the average fission time. For that, the Langevin equation was solved numerically. At each time step, a fraction of neutron is evaporated, as it is usually done. ${ }^{10}$

Again, as in the toy model, we have supposed that the binding energy of the neutron is always the same, $B_{n}=6 \mathrm{MeV}$ and that the potential is also always the same. We have also supposed that the level density parameter neither depend on deformation nor on the excitation energy.

The results are shown in Fig. 8. As in the pure fission case, the average fission time including neutron evaporation is far longer with a double humped potential than with a simple one. The rate between these two times appears to be longer with evaporation. When we decrease the excitation energy, the enlargement factor decreases. The lower limit is naturally 3 like for the pure fission process. 
This study is still very preliminary and indicates that we cannot forget the potential structures to evaluate the fission time of the SHE. But the enlargement factor seems to be too small to explain the experimental statistics on the long fission time of the $Z=120$ and 124 elements. The shell correction energy of Møller and $\mathrm{Nix}^{17}$ is too small for these two elements.

\section{Conclusions}

Crystal blocking techniques applied to measure the fission time of SHE have shown that some long fission-time components exist for the $Z=120$ and 124 elements. ${ }^{1,2,3}$ By long fission time, the authors means that $10 \%$ of the capture events have a fission time longer than $10^{-18} \mathrm{~s}$. There is no hint for such long times for the $Z=114$ element, which means that the statistics of long time events is not large enough to be observed experimentally.

We have shown that the long fission time tail is due to a competition with neutron evaporation. Then, to observe long fission time events, the effective fission barrier should be of the same magnitude as the neutron binding energy at the beginning of the evaporation chain. In the SHE region, the fission barrier is mainly due to the shell correction energy. Using the mass table of Møller and $\mathrm{Nix}^{17}$, the statistic of the long fission time component obtained by the KEWPIE2 code is far smaller than what is observed experimentally. Such a result hints that the shell correction energy of this table is too small for the $Z=120$ and 124 elements.

Such a tendency goes in the opposite direction to what is obtained from the SHE residue cross sections. Fitting the fusion models on the experimental data, the cross sections of the residue obtained by cold and hot fusion are 2 to 3 orders of magnitude larger than what is measured. For the $Z=107$ to 116 elements, the shell correction energy of the Møller and Nix table is therefore too large. ${ }^{19,20}$

The confrontation of the results of KEWPIE2 for residues and for fission times indicates that the magicity would be shifted to the $Z=120$ or 124 region. It is very difficult to give more quantitative conclusions regarding the shell correction energy. For the residue cross section, the results depend on the quality of the fusion cross sections. If these data are over estimated, the shell correction energies should not be reduced that much to fit the residue cross sections.

On the other hand, we have shown that isomeric states can enlarge quite a lot the average fission time. The conclusions on the amplitude of the increase of the shell correction energy for the $Z=120$ and 124 elements depend on this effect that is not yet completely studied. Work is still under progress.

\section{Acknowledgements}

The authors are grateful to M. Morjean for providing us preliminary experimental results and for helpful discussions. DB thanks the organizers of the workshop for their kind invitation and acknowledges useful comments of W. Ye during the workshop. He also thanks the RCNP where this paper was written for its support and 
hospitallity. D.W., Y.L. and F.G. thank the GANIL for the hospitallity during their internship. YA acknowledges a support from the JSPS grant number 18540268 and the JSPS core-to-core program on exotic femto-systems.

\section{References}

1. D. Jacquet et al, contribution to the Fusion 06 conference (2006) 239

2. A. Drouart et al, Proceedings of the Int. Symposium on exotic nuclei (Peterhof) (2004) 192

3. M. Morjean et al, Eur. Phys. J. D45 (2007) 27

4. B. Bouriquet, Y. Abe and D. Boilley, Comp. Phys. Comm. 159 (2004) 1

5. A. Marchix, Y. Abe and D. Boilley, in preparation

6. P. Langevin, Comptes Rendus de l'Acad. des Sciences Paris 146 (1908) 530

7. G. Klein, Proc. R. Soc. London A211 (1952) 431

8. H. A. Kramers, Physica VII, 4 (1940) 284

9. D. Hilscher and H. Roßner, Ann. Phys. Fr. 17 (1992) 471

10. Y. Abe, S. Ayik, P.-G. Reinhard and E. Suraud, Phys. Rep. 275 (1996) 49

11. P. Fröbrich and I.I. Gontchar, Phys. Rep. 292 (1998) 131

12. H. Bateman, Proc. Cambridge Phil. Soc. 16 (1910) 423

13. N. Bohr and Wheeler, Phys. Rev. 56 (1939) 426

14. V. Strutinsky, Nucl. Phys. 95 (1967) 420

15. V. Weisskopf, Phys. Rev. 52, (1937) 295

16. S. Hassani and P. Grangé, Phys. Lett. 137B (1984) 281

17. P. Møller and Nix, At. Data and Nucl. Tables 59 (1995) 185

18. Ignatyuk et al, Yad. Fiz. 21 (1975) 154

19. Y. Abe et al, contribution to this workshop

20. A. Marchix, PhD thesis, Université de Caen Basse-Normandie and GANIL, november 2007.

21. F. Goldenbaum et al, Phys. Rev. Let. 82 (1999) 5012

22. D. Boilley, A. Marchix, B. Jurado and K.-H. Schmidt, Eur. Phys. J. A33 (2007) 47

23. W. J. Świątecki, K. Siwek-Wilczyńska and J. Wilczyński, Phys. Rev. C71 (2005) 014602

24. D. Boilley, B. Jurado and C. Schmitt, Phys. Rev. E70 (2004) 056129

25. A. N. Malakhov, Chaos 7, 488 (1997) 


\section{$\mathrm{Bn}=6 \mathrm{MeV} \mathrm{E}^{\star}=80 \mathrm{MeV}$}

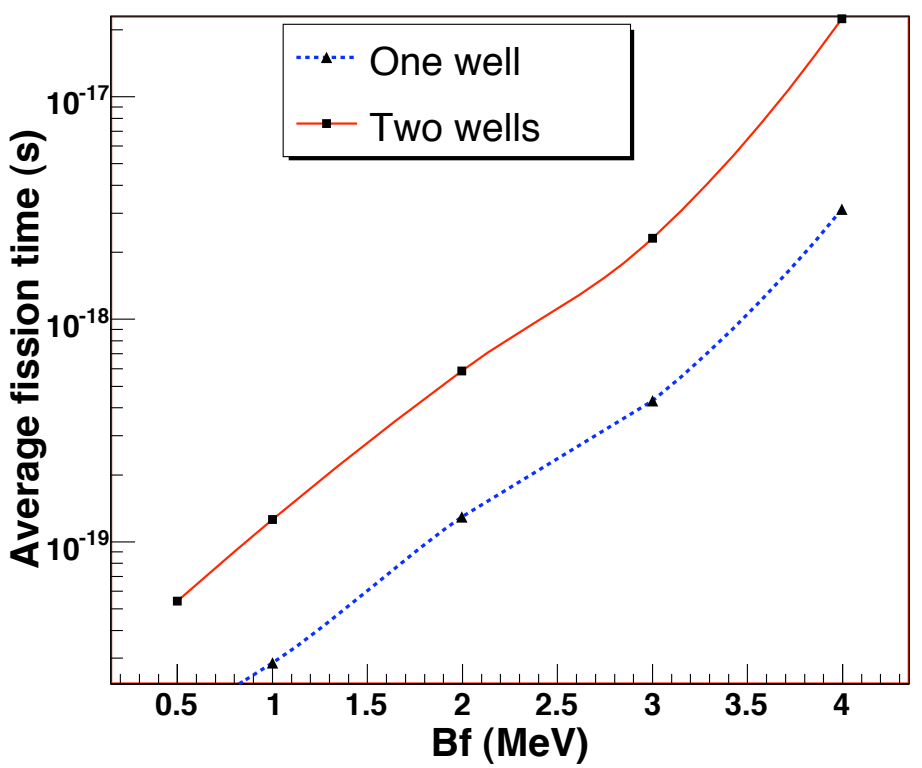

$\mathrm{Bn}=6 \mathrm{MeV} \mathrm{E}^{*}=80 \mathrm{MeV}$

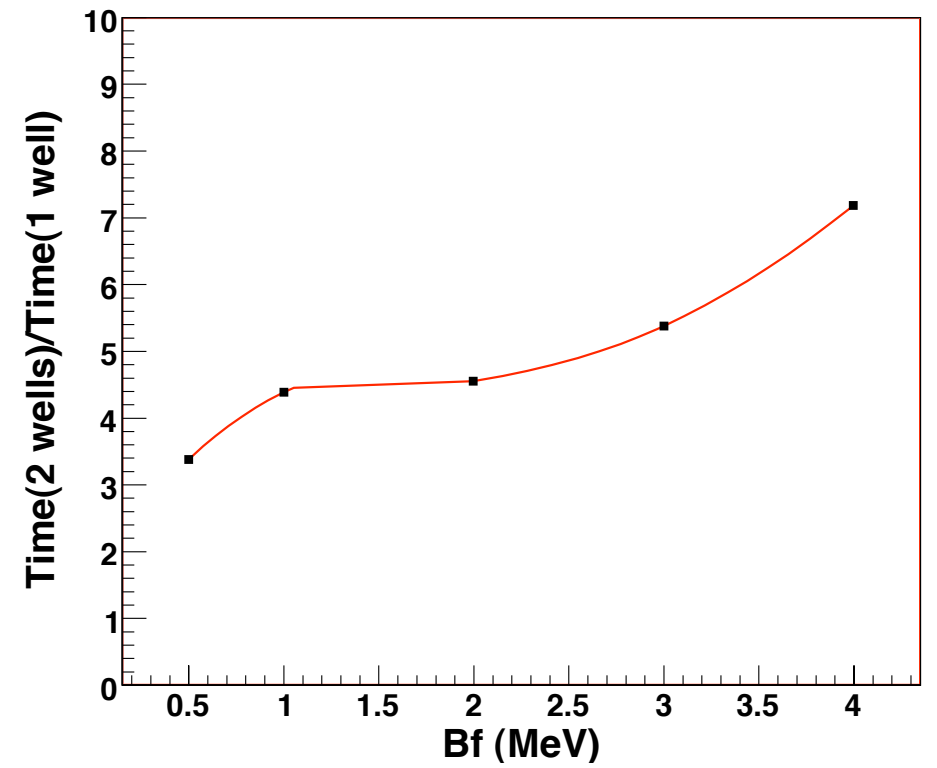

Fig. 8. Top: Average fission time calculated numerically for a simple and double-humped potential as shown in Fig. 7 including the neutron evaporation. The excitation energy is $E^{*}=80 \mathrm{MeV}$. Bottom: Ratio of the average fission time calculated with a double-humped potential over the average fission time calculated with a simple humped potential. 